\title{
Correlation and response in a driven dissipative model
}

\author{
Dana Levanony and Dov Levine \\ Department of Physics, Technion, Haifa 32000, Israel
}

(Dated: November 13, 2018)

\begin{abstract}
We consider a simple dissipative system with spatial structure in contact with a heat bath. The system always exhibits correlations except in the cases of zero and maximal dissipation. We explicitly calculate the correlation function and the nonlocal response function of the system and show that they have the same spatial dependence. Finally, we examine heat transfer in the model, which agrees qualitatively with simulations of vibrated granular gases.

PACS numbers: 05.40.a, 44.10.+i, 45.70.-n
\end{abstract}

Driven dissipative systems (DDS) occur in many different contexts, from collections of macroscopic particles to biological systems. Such systems are intrinsically out of equilibrium, and need the input of energy in order to remain functional. One prototype DDS is a granular gas [1], a collection of inelastic grains which dissipate energy through collisions; these have been examined extensively, both experimentally [2, 3] , numerically [4, 5, [6], and analytically [7]. Because of the difficulty in treating granular gasses analytically, stochastic mean-field models have been studied. One such is the Maxwell model [8], which assumes a velocity independent collision rate and no spatial structure; this facilitates analytical calculation [9] of quantities such as the velocity distribution function and its moments.

The introduction of spatial dependence complicates and enriches the behavior, and may lead to correlations: for example, actual granular gasses exhibit spatial clustering and velocity correlations because of the dissipative collisions, as is seen in simulational studies [10]. Williams and MacKintosh 11] have shown numerically that correlations exist in a 1D driven dissipative gas provided the restitution coefficient is different from one, and Soto, et al[12] have used the BBGKY hierarchy to study the appearance of velocity correlations in inelastic hardsphere systems. Baldassarri, et al [13], and Ben-Naim and Krapivsky 14] consider a lattice variant of the Maxwell model which they solve in the freely cooling case (no driving); the latter authors calculate the spatially dependent velocity correlations which exhibit gaussian decay with distance.

In this Rapid Communication we study a model of a driven system with spatial structure: the constituent "particles" are constrained to lie on a 1D lattice with nearest-neighbor coupling. Our main goal will be to understand the connection between the system's dissipative nature and spatial correlations. The system is coupled to a heat reservoir at temperature $T$, and the model is chosen so that it has a well-defined equilibrium limit for certain values of the system parameters. The meanfield version of the model, which has no spatial structure, can be solved exactly [15], in the sense that all the moments of the energy distribution may be calculated. For the model of this Rapid Communication, we calculate the two-point correlations of the system analytically, and demonstrate that non-zero correlations always exist except for the cases (a) in which there is no dissipation (in agreement with the results of $[1,16]$ ) or (b) when the dissipation is maximal. For dissipative systems driven by thermal contact with a heat bath, it is not only the bath temperature which determines the steady-state of the system; the nature of the coupling to the bath is relevant as well (in contrast to non-dissipative systems, for which this last plays no role in the determination of the equilibrium state). With this in mind, we calculate the response of the system to a change in one of these defining parameters, and compare the spatial dependence of the nonlocal response function to that of the correlation function. Finally, motivated by the question of energy flow in vibrated granular materials [4, 5, 17] we study how the internal dissipation affects the nature of heat transfer, and calculate the energy along a chain whose ends are in contact with a heat bath. We note that the model studied in reference [18] in this context is the same as our model in the absence of dissipation.

The system we study is a generalization of a model introduced in 15], which can be regarded as the mean-field version of the case of this Rapid Communication. We consider $\mathrm{N}$ particles localized on sites $n$ of a 1D lattice, each characterized by its energy $E_{n}$. The entire chain is coupled to an external Boltzmann-distributed bath with temperature T. At each time step an interaction occurs in the system, either between system particles or with the external bath. Specifically, a particle is chosen randomly and its interaction follows the stochastic rule :

$$
E_{n}(t+d t)=\left\{\begin{array}{cc}
\frac{\text { value }}{E_{n}(t)} & \frac{\text { probability }:}{1-\Gamma d t} \\
z \alpha\left(E_{n}(t)+E_{n+1}(t)\right) & \frac{1}{2}(1-f) \Gamma d t \\
z \alpha\left(E_{n}(t)+E_{n-1}(t)\right) & \frac{1}{2}(1-f) \Gamma d t \\
z\left(E_{n}(t)+E_{B}\right) & f \Gamma d t
\end{array}\right.
$$

Here $\Gamma$ is the overall rate of interaction of a particle; it sets the time scale and is irrelevant to the steady state. $f$, the strength of the coupling to the bath, is a constant which determines the probability of a particle interacting with the bath, $\alpha \in[0,1]$ is a parameter characterizing 
the dissipation in an interaction (in analogy to a restitution coefficient), $z$ is a stochastic variable uniformly distributed between 0 and 1 ; and $E_{B}$ is the energy of a particle chosen randomly from the bath. In what follows, we shall only be interested in the steady states of the system.

Our main results are: (i) correlations appear in this system for all $0<f<1$ provided $\alpha<1$; when $\mathrm{f}=0,1$ or $\alpha=1$ there are no correlations; (ii) the spatial decay of the correlation function is calculated and found to be exponential in the limit of $N \rightarrow \infty$; (iii) the nonlocal response function (to a localized change in $f$ or $T$ ) is proportional to the correlation function. This last point is reminiscent of the fluctuation-dissipation theorem of equilibrium statistical mechanics, but in our case there is no a priori reason to expect that the two functions will exhibit the same spatial dependence.

\section{CORRELATION AND RESPONSE}

Using the dynamics presented in (11) we can write equations describing the time evolution of the moments of the energy distribution and the correlation functions in the system. We will be interested in the steady-state values of the moments, so we set the time derivatives to zero. It is simple to show that the average energy is given by $\overline{E_{n}} \equiv \bar{E}=\frac{T f}{2-f-2 \alpha(1-f)}$, with $T$ being the bath temperature in units where the Boltzmann constant is unity. In order to compute the correlation function $C(n, n+k) \equiv \overline{E_{n} E_{n+k}}-\bar{E}^{2}$, we need to calculate the second moments; this leads to a set of coupled equations:

$$
\begin{gathered}
\overline{E^{2}}=\frac{2 \alpha^{2}(1-f) \overline{E_{i} E_{i+1}}+2 T f \bar{E}+2 f T^{2}}{3-2 \alpha^{2}(1-f)-f} \\
\overline{E_{n} E_{n+1}}=\frac{6 f T \bar{E}+(1-f) \alpha\left(\alpha \overline{E^{2}}+3 \overline{E_{n} E_{n+2}}\right)}{9-3 f-\alpha(1-f)(3+\alpha)} \\
\overline{E_{n} E_{n+k}}=\frac{\frac{1}{2} \alpha(1-f)\left(\overline{E_{n} E_{n+k+1}}+\overline{E_{n} E_{n+k-1}}\right)+f T \bar{E}}{2-\alpha(1-f)-f} .
\end{gathered}
$$

In the last equation, $k \geq 2$. We note that if $f=0,1$ or $\alpha=1$, then $C(n, n+k)=0$ : there are no correlations in these cases. This is consistent with the two-dimensional granular gas simulation of [16] where velocity correlations disappear as the restitution coefficient goes to 1 , and reminiscent of similar behavior of the density correlations [1]. The case $\alpha=0$ is unique in the sense that we obtain the mean field result for the distribution function and all correlations disappear except $C(n, n+1)$.

For general $f$ and $\alpha$, the above coupled equations can be written as a matrix equation of the form $\mathcal{A} \vec{W}=\vec{V}$, where $\vec{W}$ is a vector whose $k^{t h}$ place is $\overline{E_{n} E_{n+k}}$, and
$\vec{V}$ is a vector of constants. The $N \times N$ matrix $\mathcal{A}$ may be decomposed as $\mathcal{A}=\mathcal{T}-\mathcal{B}$, where $\mathcal{T}$ is a tridiagonal matrix with constant diagonals (which can be inverted with the help of [19]), and $\mathcal{B}$ is an $N \times N$ matrix which is zero everywhere but the upper left $3 \times 3$ block (which is denoted by the $3 \times 3$ matrix $\mathcal{G}$ ). $\mathcal{A}^{-1}$ may be computed from the relation 20 .

$$
\mathcal{A}^{-1}=\mathcal{T}^{-1}+\mathcal{T}^{-1} \mathcal{B} \mathcal{Q} \mathcal{T}^{-1}
$$

where $\mathcal{Q}$ is an $N \times N$ matrix of zeros except for the upper left $3 \times 3$ block which is the matrix $(\mathcal{I}-\mathcal{G})^{-1}, \mathcal{I}$ being the $3 \times 3$ unit matrix. In the limit $N \rightarrow \infty$ this yields the result that the correlations decay as

$$
C(n, n+k)=D(\alpha, f) e^{-\frac{k}{\lambda}}
$$

where $D(\alpha, f)$ is a continuous function, and $\lambda$ is the correlation length, given by $\lambda^{-1}=\operatorname{arccosh} \frac{2-\alpha(1-f)-f}{\alpha(1-f)}$.

We note that the correlation length diverges as $\alpha \rightarrow 1$ and $f \rightarrow 0$, although we know that for $\alpha=1$ there are no correlations; this is because $D(\alpha, f) \rightarrow 0$ for these values. This means that around $\alpha=1$ and $f=0$ the correlations are the longest-ranged but the weakest. The behavior of the correlation length as a function of $f$ and $\alpha$ is shown in Figure 11 in which results from simulating the model are presented for comparison.

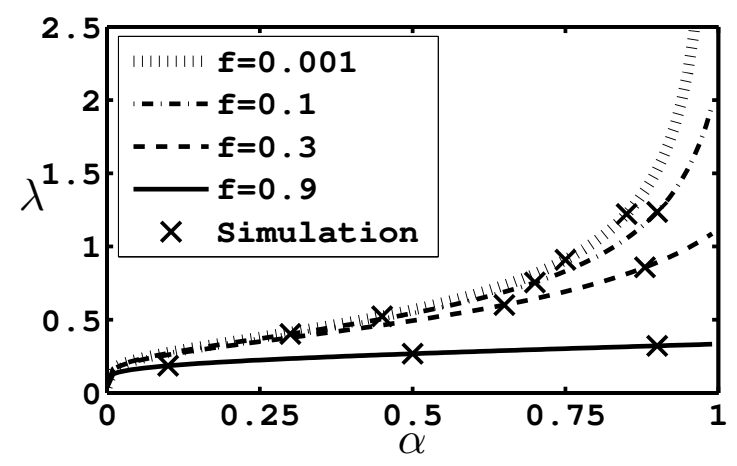

FIG. 1: The correlation length as a function of $\alpha$ for different values of $f$.

Because of the correlations, the single-particle energy distribution function $P(E)$ cannot be calculated analytically for general $\alpha$. For $\alpha=1$, detailed-balance holds, and an H-theorem may be proved, and the system comes to thermal equilibrium at the bath temperature $T$. When $\alpha=0$, the generating function $g(\omega)$ (defined by $g(\omega) \equiv\left\langle e^{-\omega E}\right\rangle=\int_{0}^{\infty} e^{-\omega E} P(E) d E$ ) is the same as that of the mean field model [15]: $g(\omega)=$ $(\omega T+1)_{2} F_{1}(1,2,2-f,-\omega T)$ where ${ }_{2} F_{1}$ is a hypergeometric function.

For $f=0$ (and $\alpha \neq 1$ ) the system energy decays to zero and therefore $P(E)=\delta(0)$ is the trivial steady-state. In Figure 2 we plot the energy distribution function $P(E)$ 
for different values of $\alpha$. We note that the distributions in Figure 2] are reminiscent of those found for the mean-field case $[15]$.

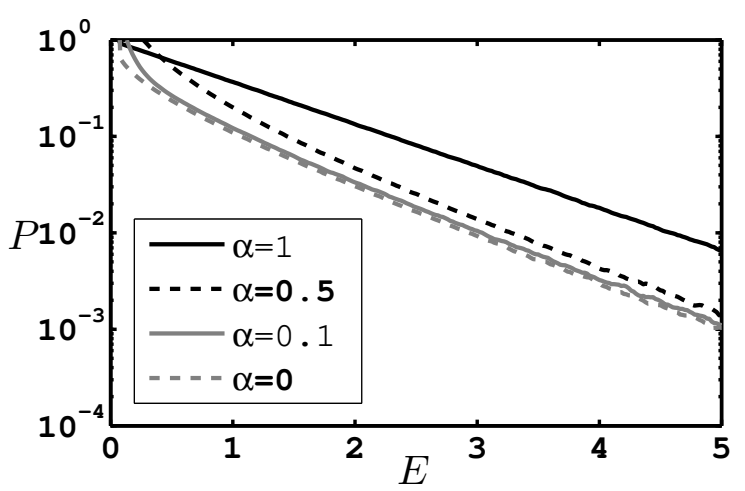

FIG. 2: The distribution function on a logarithmic scale as found from Monte Carlo simulation with $f=0.5$ and $T=1$.

For a dissipative system coupled to a bath, both the coupling strength $f$ and the bath temperature $T$ determine the behavior of the system. We will now consider the response of the system to a local change in the coupling to the bath. We stress that this is not a measurement which can be performed on a system in equilibrium, for which $f$ plays no role in the steady state, and it is thus intrinsically a non-equilibrium measurement. Nevertheless, we shall see that, reminiscent of fluctuationdissipation relations, the spatial response to such a perturbation is proportional to the spatial correlation function.

We imagine that each site in the system is coupled to a Boltzmann-distributed bath at temperature $T$ with a coupling strength $f$. We seek to calculate the change of energy at site $n+k$ due to small change in $f$ (say, from $f$ to $f_{0}$ ) at location $n$. Having changed the coupling at site $n$, the system no longer has spatial invariance, so instead of writing the time-evolution equations for a single particle, we must consider the dynamics of the entire chain. The structure of the resulting equation 20] is again of the matrix form $\vec{A} \vec{E}=\vec{V}$, where $\vec{E}$ is a vector of the energies $\left\{E_{j}\right\}$, and $\vec{V}$ is a vector of constants related to the boundary conditions. $\mathcal{A}$ is a matrix which depends on $\alpha, f$ and $f_{0}$ which can be inverted to yield $\bar{E}_{n+k}$, from which we obtain that, in the $N \rightarrow \infty$ limit,

$$
\left(\frac{d \bar{E}_{n+k}}{d f_{0}}\right)_{f_{0}=f}=B(\alpha, f) e^{-k / \lambda}
$$

where $B(\alpha, f)$ is a continuous function, and where $\lambda$ has the same value as in Equation 3 This describes the nonlocal response of the system at a site a distance $k$ away from the point of a local change in coupling to the bath. We note that this intrinsically non-equilibrium response has the same spatial decay as correlation function.

We may similarly ask what the response at a site $n+k$ is to a local change in temperature $T$ at site $n$. As before, we are able to invert the resulting matrix equation, which, in the limit $N \rightarrow \infty$ [20] gives:

$$
\frac{d \bar{E}_{n+k}}{d T_{n}}=\frac{f}{\alpha(1-f) \sinh \left(\lambda^{-1}\right)} e^{-k / \lambda}
$$

In an equilibrium system, the response of an observable to a small change in its conjugate field is proportional to the correlations of this variable, with the system's temperature being the constant of proportionality. Although our response measurements do not take this form, it is interesting to note that in both cases, the response of the energy $\bar{E}_{n+k}$ to a local change in the bath interaction at site $n$ is proportional to the correlation function $C_{n, n+k}$. Moreover, for given bath temperature, the ratios of the response to the correlation (for both types of response) decrease monotonically with $\alpha$, and diverge as $\alpha \rightarrow 0$.

\section{HEAT TRANSFER}

We have thus far considered a system all of whose sites are in contact with the same external bath. As an application of the ideas of the previous section, we now turn our attention to the case where the ends of the lattice are coupled to heat baths at temperatures $T_{-}$and $T_{+}$, respectively. For the case of $\alpha=1$, we expect that the temperature profile (i.e. the average energy at a site) will be a linear interpolation between the two wall temperatures; this is a manifestation of the Fourier law 17, 18, 21, 22].

The case where $\alpha \neq 1$ may be thought of as an idealization of energy transport through an inhomogeneous driven granular gas, where we might imagine causing walls to vibrate and ask how this is manifested throughout the system. For example, Herbst, et al [5] and Barrat, et al 4] treat a collection of inelastic disks in 2D held between vibrating walls, and find a characteristic decay of the granular temperature with distance from the wall. We modify our system so that of the particles on the lattice, only those at the end $(k=0, N+1)$ are coupled to baths, at temperatures $T_{-}$and $T_{+}$, respectively. For all other particles, the dynamics are as in Equation [1 with $f=0$. In similar fashion to the previous calculation, the relation for the average energy at site $k$ may be expressed as a matrix equation, which may be inverted to give

$$
\bar{E}_{k}=\frac{T_{+} \sinh [(k-N-1) \eta]+T_{-} \sinh [k \eta]}{\sinh [(N+1) \eta]}
$$

with $\eta=\operatorname{arccosh} \frac{2-\alpha}{\alpha}$. This result is plotted in Figure 3. We note that for $\alpha \rightarrow 1$, the profile is linear, as expected [18]. For all other $\alpha$, sufficiently far from a wall the decay is exponential with decay length $\eta$. It is interesting to note the similarity of these results to those of the numerical study of driven granular gasses in 4, 5]. 
This result may be understood in the context of the Fourier law 22] which relates the rate of heat flow, $\frac{d Q}{d t}$ to the local temperature gradient: $\frac{d Q}{d t}=-\kappa \frac{d^{2} T}{d x^{2}}$. In our case, the energy $E_{i}$ plays the role of both $Q$ and $T$, and its time evolution is governed by:

$$
\begin{aligned}
\frac{d E_{i}(t)}{\Gamma d t}= & \frac{2 E_{i}(\alpha-1)}{N+1}+\underbrace{\frac{\alpha}{2(N+1)}\left(E_{i+1}-E_{i}\right)}_{J_{+}} \\
& -\underbrace{\frac{\alpha}{2(N+1)}\left(E_{i}-E_{i-1}\right)}_{J_{-}}
\end{aligned}
$$

The terms on the right hand side of this equation can

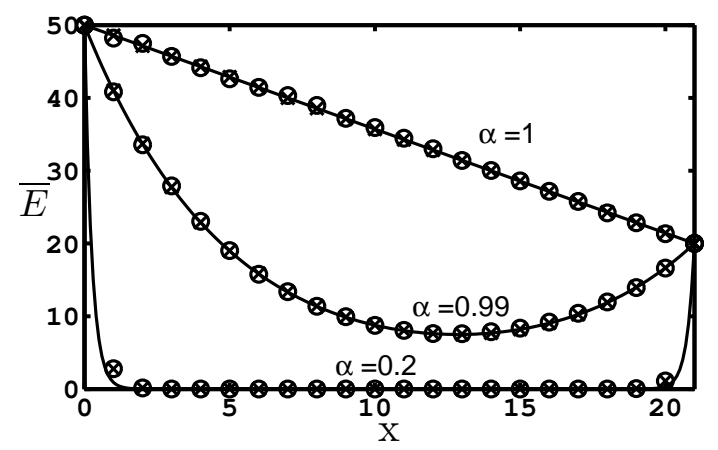

FIG. 3: Average energy as a function of position for a 20 particle lattice for different values of $\alpha$. The symbols $\circ$ are from a simulation of the model, the values from - from Equation 6 and the continuous curve is the solution of the damped diffusion equation obtained in the continuum limit.

be interpreted as a sink term, and the heat flow out of, and into site $i$ respectively. The net average energy flux into $i$ is $J_{-}=-\kappa\left(\overline{E_{i}}-\overline{E_{i-1}}\right)$ with heat conductivity coefficient $\kappa=\frac{\alpha}{2(N+1)}$. We note that in the continuum limit we obtain a damped diffusion equation for the steady-state: $\alpha \nabla^{2} E+4(1-\alpha) E=0$ with boundary condition $E(x=0)=\alpha T_{+}, E(x=N+1)=\alpha T_{-}$.

In this Rapid Communication, we have described exact calculations for the spatial dependence of correlations and response for a model DDS. It is intriguing that, despite the fact that the fluctuation-dissipation theorem is not applicable to this system, the nonlocal response to a change in temperature or bath coupling has the same spatial dependence as the correlation function. Of course, it remains to be seen what the nature of the temporal behavior of these functions is, and it would be surprising if they were to exhibit the same frequency dependence.

We would like to thank Joseph Avron, Guy Bunin, Jean-Pierre Eckmann, Joshua Feinberg, Michael Fisher, Oded Kenneth, Yair Srebro, and Annette Zippelius for interesting and enlightening discussions. D. Levine gratefully acknowledges funding from Israel Science Foundation grants 88/02 and 660/05, and the Technion Fund for the Promotion of Research, and thanks the New York University Physics department for its hospitality.

[1] Granular Gases, edited by T. Pöschel and S. Luding, (Springer, Berlin, 2001); Granular Gas Dynamics, edited by T. Pöschel and N. Brilliantov, published as Lecture Notes in Physics 624 (2003).

[2] A. Kudrolli, M. Wolpert, and J.P. Gollub, Phys. Rev. Lett. 78, 1383 (1997).

[3] F. Rouyer and N. Menon, Phys. Rev. Lett. 85, 3676 (2000); K. Feitosa and N. Menon, Phys. Rev. Lett. 88, 198301 (2002).

[4] A. Barrat, E. Trizac, Phys. Rev. E 66, 051303 (2002).

[5] O. Herbst, P. Müller, M. Otto and A. Zippelius, Phys. Rev. E 70, 051313 (2004).

[6] A. Puglisi, A. Baldassarri, and V. Loreto, Phys. Rev. E 66, 061305 (2002).

[7] T.P.C van Noije and M.H Ernst Granular matter 1, 5764 (1998); M.H. Ernst and R. Brito, Phys. Rev. E 65, 040301(R); I. Goldhirsch, S.H. Noskowicz and O. BarLev, Phys. Rev. Lett. 95, 068002 (2005); I. Goldhirsch and N. Sela, Phys. Rev. E 54, 4458 (1996).

[8] D. ben-Avraham, E. Ben-Naim, K.Lindenberg and A.Rosas, Phys. Rev. E 68, 050103(R) (2003); A. Santos and M.H. Ernst, Phys. Rev. E 68, 011305 (2003).

[9] E. Ben-Naim and P.L. Krapivsky, Phys. Rev. E 61, R5 (2000); E. Ben-Naim and P.L. Krapivsky, Phys. Rev. E 66, 011309 (2002).

[10] I Goldhirsch and G. Zanetti, Phys. Rev. Lett. 70, 1619 (1993); M.R. Swift, M.Boamfa, S.J.Cornell and A.Maritan, Phys. Rev. Lett. 804410 (1998); S. Luding, R. Cafiero, H.J. Herrmann, Lecture Notes in Physics 624, 293 (2003).

[11] D.R.M. Williams, F.C. MacKintosh, Phys. Rev. E 54, R9 (1996).

[12] R. Soto, J. Piasecki and M. Mareschal, Phys. Rev. E 64 031306 (2001); R.Soto and M.Mareschal, Phys. Rev. E 63, 041303 (2001).

[13] A. Baldassarri, U. Marini Bettolo Marconi, and A. Puglisi, Europhys. Lett. 5814 (2002).

[14] E. Ben-Naim and P.L. Krapivsky, Lecture Notes in Physics 624, 65 (2003).

[15] Y. Srebro and D. Levine, Phys. Rev. Lett. 93, 240601 (2004).

[16] G. Peng and T. Ohta, Phys. Rev. E 58, 4737 (1998).

[17] R. Soto, M. Mareschal and D.Risso, Phys. Rev. Lett. 83 5003 (1999).

[18] C. Kipnis , C. Marchioro and E. Presutti, J. Stat. Phys. 27, 65 (1982).

[19] G.Y. Hu and R.F. O'Connell, J. Phys. A 29 1511-1513 (1996).

[20] D. Levanony and D. Levine, to be published.

[21] A.V. Savin, G.P. Tsironis and A.V. Zolotaryuk, Phys. Rev. Lett. 88, 154301 (2002); B. Hu, B. Li and H. Zhao, Phys. Rev. E 57, 2992 (1998).

[22] F. Bonetto, J.L. Lebowitz and L. Rey-Bellet, Mathematical Physics 2000, Edited by A. Fokas, A. Grigoryan, T. Kibble and B. Zegarlinsky, Imprial College Press, 128151 (2000). 